http://jmscr.igmpublication.org/home/ ISSN (e)-2347-176x ISSN (p) 2455-0450

crossref DOI: https://dx.doi.org/10.18535/jmscr/v7i9.20

\title{
A Clinical Study on Correlation of Tympanic Membrane Abnormalities with Audiometric and Tympanometric Findings
}

\author{
Authors \\ Dr Shenny Bhatia', Dr Manpreet Singh Nanda², Dr Mohit Goyal ${ }^{3}$, \\ Dr Vipan Gupta ${ }^{4}$, Mrs Deepika Sharma ${ }^{5^{*}}$ \\ ${ }^{1}$ Associate Professor, Department of Otolaryngology, MMMC\&H, Solan \\ ${ }^{2}$ Professor, Deptt of Otolaryngology, MMMC\&H, Solan \\ ${ }^{3}$ Senior Resident, Deptt of Otolaryngology, MMMMC\&H, Solan \\ ${ }^{4}$ Professor and HOD, Department of Otolaryngology, MMMC\&H, Solan \\ ${ }^{5}$ Audiologist, Deptt of Otolaryngology, MMMC\&H, Solan \\ *Corresponding Author \\ Mrs Deepika Sharma \\ Audiologist, Deptt of Otolaryngology, MMMC\&H, Solan, India
}

\begin{abstract}
Various Tympanic membrane conditions are known to affect the stiffness and mass component of the ear differently affecting hearing level. Thus the present study was conducted to find out the correlation of tympanic membrane abnormalities with the audiometric and tympanometric findings.

Material \& Methods: All the patients reporting to the E.N.T Deptt. in the age range of 11-70 years with ear problems were examined. Total 111 ears were taken in the study. Inclusion criteria: The patients with tympanic membrane abnormalities e.g myringitis, Myringosclerosis, tympanic membrane atrophy and retracted tympanic membrane, Granular TM were examined under microscope and audiometery and tympanometry was done.

Results: In the present study the most common Tympanic membrane abnormality seen was Retraction. Among which the Grade I retraction was the commonest. Second most common was Tympaosclerosis. It was noticed that among retraction, type A graph was commonest. In tympanosclerotic patch As ,atrophy Ad was commonest while in SOM Cases B type was commonest.There was significant correlation between hearing loss and Grade of retraction but no significant correlation was observed in Pure tone audiometry and typanometry.

Conclusion: In our study we observed that TM abnormality is not always associated with significant audiological findings.
\end{abstract}

\section{Introduction}

In standard Indian otorhinolyringologic settings otoscopy or otomicroscopy and audiological tools are used to diagnose various middle ear pathologies. Tympanometry provides useful information about the presence of fluid in the middle ear, mobility of middle ear system and ear canal volume. It has been recommended in conjunction with more qualitative information like history, appearance and mobility of Tympanic membrane (TM). Middle ear pathologies are known to affect the stiffness and mass component 
of the ear differently affecting tympanometric findings and hearing level. Isolated TM abnormalities may result as sequelae of otitis media or otherwise.

A healthy tympanic membrane has three layers, outer epithelial layer, inner mucosal layer and middle fibrous layer. How these three Tympanic layers are affected by the pathology determines the impact on conduction of sound and the stiffness and mass component in audio logical findings.

In atrophic TM the fibrous layer is deficient or totally absent due to thinning of lamina propria ${ }^{[1]}$. Tympanosclerosis is the hyaline degeneration of the connective tissue within the middle layer of $\mathrm{TM}^{[2]}$. The etiology is basically unknown, however various hypothesis exists. Some of them are as sequelae of severe or acute otitis media, bacterial or viral ${ }^{[3]}$. The role of osteopontin or the role of HLA -antigens HLA-B35 and HLA DR3 may play an important role ${ }^{[4]}$

Granular tympanic membrane is a chronic disorder, is characterized by lateral squamous deepithilization and granulation of TM. Chronic myringitis is usually loss of tympanic membrane epithelium without disease within tympanic cavity.

Tympanic membrane retraction is pathological invagination of TM into middle ear space and is usually the sequelae of otitis media with effusion. Prolonged dysfunction of eustachian tube and excessive negative pressure may lead to atrophic changes in middle and fibrous layer of TM leading to localized or generalized TM atelectasis. ${ }^{[5]}$. Retraction pockets can be organized in anatomically defined hierarchical grading system of pars tensa retraction from grade I to grade IV according to Sade's classification.

Though plethora of studies are done in the past literature relating middle ear pathologies with the tympanometeric findings, limited data could be found correlating various isolated tympanic membrane abnormalities with the impedance and audiometric findings.
Li et al studied TM retraction, Hearing loss and multifrequency tympanometry. $\mathrm{He}$ reported positive correlation between retraction severity and hearing level ${ }^{[6]}$. Holt et al demonstrated that isolated abnormalities of TM without middle ear disease can alter the tympanogram and the type of tympanogram can be predicted from the nature and extent of abnormality ${ }^{[7]}$. M Sunita reported that most patients with Atrophic TM have normal hearing ${ }^{[8]}$. Indranil pal illustrated the clinicopathological and audiological findings in tympanosclerotic tympanic membrane and found that it is associated with a abnormal tympanogram indicating stiffness of sound mechanism ${ }^{[4]}$. Bhushan did a comparative study of Eustachian tube function in normal and retracted tympanic membrane ears with tympanometry and video nasophayryngoscopy. Their study revealed that most patients had grade I retraction. $60 \%$ of patients with grade I retraction had A type tympanometric curve without any hearing loss. ${ }^{[9]}$

This article discusses correlation of tympanic membrane abnormalities with audiometric and tympanometric findings. A review of Microscopic Examination, Pure Tone audiometry (PTA) and Impedance is provided to illustrate the integrated use of these diagnostic tools.

The study was conducted with the following objectives

- To find clinical correlation of TM abnormalities (Graded retraction, tympanosclerosis, atrophy, granulation e.t.c) with hearing thresholds.

- To find clinical correlation of grade of retraction of pars tensa with hearing thresholds and tympanometric graph.

- To find clinical correlation of Pure tone audiometry with Tympanogram.

\section{Material and Methods}

All the patients (age range of 11-70 years) reporting to the E.N.T OPD at MMMC\&H Solan for the period of 12 months from June 2015 to June 2016 underwent otomicroscopic examination. Only the ears with tympanic 
membrane abnormalities were included in the study group. The TM abnormalities included were tympanosclerosis, atrophic tympanic membrane, Granular myringitis, retracted tympanic membrane, acute otitis media, combined TM abnormalities like retraction with tympanosclerosis and serous otitis media.

The ears with perforated tympanic membrane, patent grommet, and impacted wax were excluded from the study group. Following otomicroscopic examination the patients were made to undergo Pure tone audiometry and Tympanometry in a sound treated room. ALPS AD2100 audiometer was used for pure tone Audiometry. Air conduction and Bone Conduction thresholds at $500,1 \mathrm{~K}, 2 \mathrm{~K} 4 \mathrm{~K}$ AND $8 \mathrm{~K}$. Based on the Audiogram hearing loss was classified as Normal $<25 \mathrm{Db}$, Mild 26-40dB, Moderate 40-55Db Moderately severe 55-70, severe 70-90 db. Profound < $90 \mathrm{Db}$. Patients with Sensorinural component of hearing loss (Sudden SNHL, NIHL, Presbyacusis, Ototoxicity) or indications of otosclerosis were excluded from the study group. Interacoustics middle ear analyser was used for tympanometry .Tympanogram was evaluated with regard to the Type of Tympanogram (Jerger's classification ) .In total 111 ears were taken up for the study. Appropriate approval was procured from the institution and informed consent was taken up from the patients.

The data so obtained was recorded and analysed using discriptive statistics to determine frequencies for variables like Gender, Age, Type of Tympanograms and Audiometric findings. Statistical Analysis was done by Proportion test (z).The value of $\mathrm{p}<0.05$ was taken to be significant.

\section{Results}

The study included 54 males and 57 females and total of 111 ears. The male female ratio was approximately 1:1. Table 1 Shows gender distribution of various TM abnormalities. The age range was 11-70 years. The commonest group affected by TM abnormalities was 31-50 years.
Table 2. Shows Age wise distribution of various TM abnormalities. Table 3 shows the age wise distribution of grade of retraction. .In the present study the most common Tympanic membrane abnormality seen was Retraction $38.7 \%$. Among which the Grade I retraction was the commonest $54 \%$. Second most common was Tympaosclerosis $18 \%$. Figure 1 shows percentage of common Tympanic membrane abnormalities.Figure 2 shows percentage of various grades of retraction.

Ears with tympanosclerosis (Ts) and retraction had maximum Average hearing loss which was $30 \mathrm{~dB}$. Average Hearing loss of $27 \mathrm{~dB}$ was obtained in ears with SOM. No significant hearing loss was obtained in rest of the TM abnormalities. Figure 3 show the average hearing loss in various TM Abnormalities.

Figure 4 shows the commonest Tympanometric graphs in TM Abnormalities .Ears with retracted TM obtained various curves .Type A was the commonest $(37 \%)$ in retraction followed by $\mathrm{C}$ Type of curve (23\%). In Tympanosclerosis 60\% had As type tympanogram. $81 \%$ ears of SOM obtained B Type of curve. Atrophic TM showed Ad to be the most common curve. In ears with AOM and Granular myringitis Type A of tympanogram was most common. In retraction with tympanosclerosis we obtained Ad type of curve followed by $\mathrm{C}$.

The main aim of the study was to determine the correlation between the TM abnormalities and hearing loss. The degree of hearing loss was classified based on WHO classification. Maximum patients had normal hearing inspite of TM abnormalities. The Chi square test was used to determine the significance of the correlation. The $\mathrm{p}$ value between the TM Abnormalities and hearing loss was non significant $\mathrm{p}=0.723$ $(\mathrm{p}<0.05)$. The Chi square value was 8.76 . Indicating that there is no significant correlation between hearing loss and TM Abnormalities. Table 1 Shows the correlation between the TM abnormalities and hearing loss.

In the correlation of grade of retraction with hearing it was seen that maximum patients had 
normal hearing. The Chi square test was used to determine the significance of the correlation. The $\mathrm{p}$ valve between the Grade of retraction and hearing loss was significant $\mathrm{P}=0.001$ ( $\mathrm{p}<0.0 .05$ ) The Chi square value was 22.111 indicating that there is a significant correlation between hearing loss and Grade of retraction. Table 4 Shows the correlation between the grade of retraction and hearing loss.

The Chi square was also used to determine the correlation between the Average hearing loss and Type of tympanogram. The $\mathrm{p}$ value between the two was 0.64 which was not significant. $(\mathrm{p}<0.05)$. The Chi square value was 6.03 indicating that there is no significant correlation between hearing loss and Tympanometric curves. Table 5 shows the correlation between the Average hearing loss and Type of tympanogram.

\section{Discussion}

In our study it was observed that there was almost equal incidence of various tympanic membrane abnormalities in male and females. Thus we could not find any significant gender predilection. This is in agreement with study conducted by Indranil Pal on tympanosclerosis but not with M Sunita and Priyank D on atrophy and chronic retraction respectively ${ }^{[4,8,10]}$. Bhaya et al also had similar results ${ }^{[2]}$. The age wise distribution of various TM abnormalities showed that the incidence was most common in third to fifth decade of life which is in accordance with the study conducted by Indranil Pal on tympanosclerosis ${ }^{[4]}$ and with Priyank D on chronic retracted membranes ${ }^{[10]}$.

The retraction of Tympanic membrane was graded as per Sade's classification.

Grade I- mild retraction not touching the long process of Incus.

Grade II- Retracted drum touching the long process of Incus.

Grade III retracted drum touching the promontory. Grade IV drum plastered to the promontory.

Retraction was the most common abnormality (39\%) seen in our study which may be attributed to the hilly terrain of our region leading to frequent altitude changes. Retraction was seen commonly in third and fourth decade of life which could be due to frequent travel in this age group due to work related issues. Retraction was followed by Tympanosclerosis and least was Granular Myringitis (2.07\%).

It was seen that hearing was normal in $63 \%$ of cases while $72 \%$ had abnormal tympanogram. We observed that Type A graph was seen in $37.2 \%$ of cases of Retraction while $62.8 \%$ had other abnormal graphs. This is in accordance with study conducted by Holmquist et al ${ }^{[11]}$ and $\mathrm{B}$ Chauhan [9]. $60 \%$ cases had normal hearing in accordance with Bhushan which can be attributed that maximum cases of retraction were of grade 1 $(53.5 \%)$ with average hearing loss of $15.33 \mathrm{~dB}$. Out of 23 cases of grade 1 retraction $47 \%$ had type A tympanogram and $53 \%$ had $\mathrm{Ad}$, As \& C type of tympanogram, not in agreement with study conducted by Zheng et $\mathrm{al}^{[12]}$ where they noted type $\mathrm{B}$ and $\mathrm{C}$ were more common in cases of retraction.

$83 \%$ of Grade 1 retraction had normal hearing. In grade II we noticed that hearing was normal in only $40 \%$ of cases and only $30 \%$ had A type of graph while $70 \%$ had As type. In grade III it was observed that only $33 \%$ had normal hearing and $78 \%$ of patients had C type of graph. Our study is in accordance with study done by Chauhan where Type A was maximum in grade 1 retraction. Similarly it was observed that as the Grade of retraction progressed from I to IV the hearing thresholds increased, in accordance with Priyank D.

Tympanosclerosis was the second most common abnormality in our study. The most common type of tympanogram seen was Type As in accordance with Holt and Gibb ${ }^{[7,3]}$ but not in accordance with Indranil pal ${ }^{[9]}$. It was seen that Hearing level was normal in $65 \%$ of the cases of Tympanosclerosis. This condition does not appear to be associated with significant hearing loss, unless it involves the ossicular chain or when the sclerosis is not generalised across the effective vibrating area of the TM and is frequently associated with changes 
in the tympanogram possibly due to mass loading of the Tympanic membrane ${ }^{[13]}$.

Our study showed that atrophic TM did not have significant hearing loss. This is in agreement with study conducted by M Sunita on hearing loss. The conduction of sound is not affected in Atrophic membrane, as it does not affect the transforming mechanism in any of the ways ${ }^{[8]}$. In Accordance with the study of Holt, our study showed Ad type tympanogram to be the most common Graph. ${ }^{[7]}$ In a study done by Valtonen et al retraction was associated with mild hearing loss but tympanosclerosis and atrophy were not as seen in our study ${ }^{[14]}$.

$81 \%$ cases of SOM had abnormal Type B graph in our study which is in accordance with Tallat Najeeb et al and Khursheed Anwar ${ }^{[15,16]}$. Average hearing loss was more than $25 \mathrm{Db}$ in accordance with the study of Karan Sharma ${ }^{[17]}$.

In cases of AOM $46 \%$ had A type of tympanogram, $27.3 \%$ had $\mathrm{C}$ and $15.4 \%$ had $\mathrm{B}$ and Ad type of tympanogram. Maximum cases had normal hearing.

In the condition having both tympanosclerosis with retraction it was seen that the Average hearing loss was of $30 \mathrm{Db}$ and $80 \%$ of the cases had abnormal tympanogram. $41.7 \%$ had Ad Type of tympanogram. Combination of TM abnormalities do have hearing loss as shown in study done by Lindsay, according to him hearing loss was largely unrelated to the presence and type of abnormality. ${ }^{[18]}$.

Cases of Granular myringitis whose prevalence was least in our study in accordance with study done by Nikolas 2001 where the prevalence rate was less than $1 \%$ and showed Type A Graph to be the most common tympanometric curve with normal hearing in most of the cases as also seen in study done by Wolf $\mathrm{M}$ et al. ${ }^{[19,20]}$

There was significant correlation between the grade of retraction and hearing loss as reported by the study of Priyanka $\mathrm{D}^{[10]}$. This can be attributed to the fact that greater the Grade of retraction greater the changes in the Tympanic membrane mobility. The Grade III and IV retraction may alter the effective vibrating area of the Tympanic membrane thus affecting the hydraulic action of the transduction mechanism of the ear leading to decrease in sound transmission. Minor changes may not produce substantial hearing loss. ${ }^{[5]}$

Our study reports no significant correlation between PTA and tympanometry. This in accordance with the various other studies ${ }^{[21,22]}$ Studies have also shown that $64 \%$ of middle ear pathologies may go undetected in audiometric screening. In our study it was seen that inspite of having TM abnormalities $63 \%$ of our patients had normal hearing and only $27 \%$ had normal tympanogram.

It is seen that when TM abnormality is detected otoscopically, its presence alone or in combination can be a strong indicator of underlying middle ear pathology. However TM abnormality is not always associated with hearing loss. It was seen that minor changes in the TM did not produce substantial hearing loss as shown by the correlation between PTA and Tympanometry in our study. It is also seen that in patients with retraction pockets, hearing is not the main issue inspite of advanced disease but it is the anatomical state of the middle ear affecting the sound transforming mechanism which may result in hearing loss. These findings suggests the need for more advanced, non invasive diagnostic techniques like multifrequency tympanometry, Otoreflectance and High frequency Audiometry so that a proper picture of the effect of pathologies on the hearing mechanism can be seen. Our study needs to be explored with the above mentioned instrumentation. Further the presented study is done with smaller number of participants. Studies with larger proportion of participants with each type of tympanic abnormality and more advanced diagnostic tools needs to be done to reach a final conclusion.

\section{References}

1. Govaerts PJ, Jacobs WA Marquet J 1988 Histological study of thin replacement membrane of human tympanic membrane 
perforation. Acta Otolyryngol 105 (3-4: 297-302.)

2. Bhaya,M H,S Cha Chern, Pa Morizono T, Paparela MM.P athogenesis Of Tympanosclerosis. Otolaryngol -Head neck Surg 1993;109`;13-20

3. Gibb AG. President's Address. Tympanosclerosis. Proc R Soc Med 1976;69:155-62

4. Pal Indranil, Sengupta A Clinicopathological And Audiological Study Of Tympanosclerosis Indian Journal of otolaryngology and Head and Neck Surgery (July - September 2005) Vol 57, No 3.

5. Mierzwiński J, Fishman A.J.Retraction pockets of tympanic membrane: protocol of management and results of treatment. Otorynolaryngologia 2014, 13(2): 114121

6. Li Y, Hunter LL, Margolis RH, Levine SC, Lindgren B, Daly K, Giebink GS. Prospective study of tympanic membrane retraction, hearing loss, and multifrequency tympanometry. Otolaryngol Head Neck Surg. 1999 Nov; 121(5):514-22.

7. Holt GR, Watkins TM ,Yoder MG Assessment of tympanometry abnormalities of the tympanic membrane .Am J Otolaryngol ,1982 Mar- Apr;3(2): 112-6.

8. M Sunita. Atrophic Tympanic Membrane and Hearing Assessment. Indian $\mathbf{J}$ Otolaryngol Head Neck Surg ( Jan- March 2012) 64(1): 22-24.

9. Chauhan Bhushan, Chauhan Komal. A Comparative Study of Eustachian tube functions in Normal and Diseased Ears with Tympanometry and Videonasopharyngoscopy. Indian J Otolaryngol Head Neck Surg (December 2013) 65 ( Suppl 3):S468-S476.

10. Talati D. Priyank , Kumar R Kalpana , Ghosh Paulastya. A Comparative Study between Pure Tone Audiometry and Impedence Audiometry- Findings in Patients with Chronic Retracted Tympanic Membranes. JMSCR November 2017 Volume 05 Issue 11.

11. Holmquist J, Renvall U, Svendsen P. Eustachian tube function and retraction of the tympanic membrane. Ann Otol Rhinol Laryngol Suppl. 1980 May-Jun;89 :65-6.

12. Zheng Y, Ou Y, Yang H, Liu X, Chen $\mathrm{S}$, Clinical manifestation of attic retraction pocket Liu W.Lin Chuang Er Bi Yan Hou Ke Za Zhi. 2005 Aug;19(16):737-9.

13. Hunter L Lisa, Margolis H Robert Effects of Tympanic Membrane Abnormalities on Auditory Function J Am Acad Audiol 8: 1997 ; 431-446.

14. Valtoten HJ Qvarnberg YH ,Nuutinen J.Otological and audiological outcome five year after tympanostomy in early childhood.Larymgolscope.2002;112:669675.

15. Tallat Najeeb, Arshad Chohan,Musharaf Baig,Sayyed Ali Naqi . Frequency and Etiology of Secretory Otitis Media and its Morbidity in Children. Journal of Rawalpindi Medical College (JRMC); 2008;12(2):92-94

16. Anwar, K., Khan, S., Rehman, H. ur, Javaid, M., \& Shahabi, I. (2016). Otitis media with effusion: Accuracy of tympanometry in detecting fluid in the middle ears of children at myringotomies. Pakistan Journal of Medical Sciences,32(2), 466-470. http://doi.org/10.12669/pjms.322.9009

17. Sharma K, Mehan R, Arora A. Clinicoaudio- radiological and operative evaluation of otitis media with effusion. Indian Journal of Otology, July 2015; Vol 21 Issue 3.

18. Johnston C Lindsay, Feldman M Heidi ,Paradise L Jack et al. Tympanic Membrane Abnormalities and Hearing Levels at the Ages of 5 and 6 Years in 
Relation to Persistent Otitis Media and Tympanostomy Tube Insertion in the First 3 Years of Life : A Prospective Study Incorporating a Randomized Clinical Trial. Pediatrics July 2004 Vol 114 No 1

19. Blevins NH, Karmody CS Chronic myringitis: prevalence, presentation, and natural history. Otol Neurotol. 2001 Jan;22(1):3-10.

20. Wolf M, Primov-Fever A, Barshack I, Polack-Charcon S,Kronenberg. Granular myringitis: Incidence and clinical characteristics J Otol Neurotol. 2006 Dec;27(8):1094-7.

21. Brooks DN, Hearing Screening: A Comparative Study of an Impedance Method and Pure Tone Screening. Scandinavian Audiology Volume 2, 1973 Issue 2

22. McCandless GA, Thomas GK. Impedance audiometry as a screening procedure for middle ear disease. Trans Am Acad Ophthalmol Otolaryngol. 1974 MarApr;78(2):ORL98-102. 